\title{
Persistence, Adherence, and Risk of Discontinuation Associated with Commonly Prescribed Antihypertensive Drug Monotherapies
}

\author{
William J. Elliott, MD, PhD, Craig A. Plauschinat, PharmD, MPH, \\ Grant H. Skrepnek, PhD, MS, RPh, and Douglas Gause, MS, DrPH
}

Objective: To assess 1-year persistence and adherence with monotherapy using the most commonly dispensed individual agent in 4 antihypertensive drug classes: hydrochlorothiazide (HCTZ), amlodipine, lisinopril, or valsartan.

Design: Retrospective, longitudinal analysis of initial prescriptions during 2001 to 2002 from a nationwide administrative claims database representing 11 million covered lives in the United States.

Measurements: Drug utilization following initiation. Cox proportional hazards regression models controlled for demographics, case-mix, and concomitant treatments.

Results: Records for 60,685 subjects were included: HCTZ $(\mathrm{n}=18,713)$, amlodipine $(\mathrm{n}=11,520)$, lisinopril $(n=21,138)$, or valsartan $(n=9314)$. Over 1 year, $31 \%$ to $44 \%$ of subjects utilized no treatment for at least 60 days. Medication possession ratio (MPR) and adherence measures ranged from $73 \%$ to $90 \%$. Valsartan was associated with significantly $(P<.001)$ more favorable measures of persistence, length of therapy, time to discontinuation, MPR, and risk of discontinuation, compared with HCTZ, amlodipine, or lisinopril. The risk of discontinuation was $53 \%, 32 \%$, and $14 \%$ greater for HCTZ, amlodipine, and lisinopril, respectively, versus valsartan (all comparisons $P<.001$ ).

Conclusion: Among antihypertensive agents studied, valsartan was associated with the most favorable utilization patterns. Health care providers and systems should evaluate the use of antihypertensive drugs within their populations to identify and manage treatment discontinuation. ( $\mathrm{J}$ Am Board Fam Med 2007;20:72-80.)

Hypertension is a common and eminently treatable risk factor for major cardiovascular events, including coronary heart disease and stroke. ${ }^{1}$ Cardiovascular disease is the leading cause of morbidity and mortality in developed nations, ${ }^{2}$ and is expected to increase in worldwide importance during the next 20 to 50 years. $^{3}$ The prevalence of hypertension

This article was externally peer reviewed.

Submitted 5 June 2006; revised 12 September 2006; accepted 14 September 2006.

From the Department of Preventive Medicine, Rush Medical College at Rush University Medical Center (WJE), Chicago, IL; Novartis Pharmaceuticals Corporation (CAP, DG), East Hanover, NJ; and Center for Health Outcomes and PharmacoEconomic Research, University of Arizona College of Pharmacy (GHS), Tucson, AZ.

Funding: This work was funded by an unrestricted grant from Novartis Pharmaceuticals.

Presented previously: Preliminary findings from this investigation were presented, in part, at the American Society of Health-Systems Pharmacists Annual Meeting in December 2004.

Conflict of interest: Dr. Elliott has received grant or research support from 18 pharmaceutical companies, has worked as a consultant to 9, has delivered speeches supported by 36 , and has received "other financial or material support" from Lederle Laboratories, E. R. Lilly, Forest seems to be increasing in both developed nations, where it currently affects approximately 1 in 3 adults, ${ }^{4}$ and in developing countries. ${ }^{5}$

Meta-analyses and meta-regression analyses of clinical trials in hypertension have reported that pharmacologic drug therapy significantly reduces cardiovascular events, which is largely attributable

Pharmaceuticals, and Rhone-Poulenc Rorer, not included in the aforementioned lists of pharmaceutical companies. In addition, Dr. Elliott notes that his department at Rush Medical College has "many contracts with many pharmaceutical companies, in which I have an adjunct role, but for which I am not Principal Investigator. I suspect [the list of these companies] will look very similar to the "Manufacturer's Index" found on pages 1-16 of the 2006 Physicians' Desk Reference." Dr. Skrepnek has received funding from Pfizer and Novartis and has been a consultant to Aventis, Novartis, Bristol-Myers Squibb, and Forest Laboratories, in relation to cardiovascular products; in relation to noncardiovascular products, Dr. Skrepnek has received funding from Eli Lilly and has been a consultant to Sepracor. Drs. Plauschinat and Gause were full-time employees of Novartis Pharmaceuticals at the time this work was done.

Corresponding author: William J. Elliott, MD, PhD, Department of Preventive Medicine, Rush Medical College, 1700 West Van Buren Street, Suite 470, Chicago, IL (Email: welliott@rush.edu). 
to their blood pressure-lowering effects. ${ }^{6,7}$ As a result, current national and international guidelines focus on the importance of achieving blood pressure targets $(<140 / 90 \mathrm{~mm} \mathrm{Hg}$ for patients with uncomplicated hypertension, and $<130 / 80 \mathrm{~mm} \mathrm{Hg}$ for those with diabetes mellitus or chronic kidney disease). ${ }^{8}$ The majority of hypertensive patients in the United States ${ }^{8}$ and other countries ${ }^{9}$ do not reach these target blood pressure levels, however, partly because of poor adherence to prescribed drug therapy and lack of long-term persistence with antihypertensive therapy as assessed by pharmacy refill rates. ${ }^{9-11}$

Although all the major antihypertensive drug classes demonstrate comparable efficacy in blood pressure lowering, differences have been observed among agents in adverse-event profiles and longterm tolerability characteristics. For example, clinical trial data ${ }^{12-17}$ and observational investigations ${ }^{18-24}$ indicate that angiotensin II type 1 receptor blockers (ARBs) have fewer adverse events, improved long-term tolerability profiles, and higher adherence rates relative to other classes of antihypertensive drugs, including angiotensinconverting enzyme (ACE) inhibitors, calcium channel blockers (CCBs), beta-blockers, and diuretics. Some of the previous observational studies of adherence to different antihypertensive drugs did not include $\mathrm{ARBs},{ }^{20}$ included much smaller number of patients treated with losartan (the first ARB) than with other drug classes, ${ }^{19,21}$ or demonstrated significant inhomogeneity across different ARBs. ${ }^{24}$ This study was designed to compare persistence and adherence using the most commonly dispensed agent in each of 4 drug classes, at a time when dispensing of ARBs was relatively common.

Differential adherence to long-term therapy may have major implications for the effectiveness of antihypertensive medications in preventing cardiovascular events, ${ }^{15,25,26}$ as well as health care costs. $^{27-29}$ In a Medicaid population, continuous use of antihypertensive medications in the first year after initiation was associated with significantly lower health care costs, mainly attributable to decreased hospital expenditures. ${ }^{30}$ We conducted a study in a large patient population to assess patient persistence, adherence, and treatment discontinuation over 1 year among patients treated with the most widely prescribed antihypertensive agent in each of 4 drug classes at the time the study was conducted: thiazide-type diuretics (hydrochlorothi- azide, HCTZ); CCBs (amlodipine); ACE inhibitors (lisinopril); and ARBs (valsartan). ${ }^{31}$

\section{Methods}

This retrospective longitudinal analysis investigated a cohort of adults who received a first (index) prescription for HCTZ, amlodipine, lisinopril, or valsartan between 2001 and 2003. Prescription and other data were drawn from a Health Insurance Portability and Accountability Act (HIPAA)-compliant, de-identified administrative claims database (Thomson MEDSTAT MarketScan Research Database, Ann Arbor, Michigan), which contains combined medical and pharmacy service-level claims data for approximately 11 million covered lives enrolled in more than 100 commercial, governmental, and public health plans. The database contains comprehensive inpatient, outpatient, emergency department, pharmacy claims, prescription drug utilization, enrollment, and eligibility information.

Subjects' records were included in this analysis if they were 18 years of age or older; had continuous insurance eligibility for at least 24 months (12 months before and 12 months after the index date); had no prescription claim for a diuretic, CCB, ACE inhibitor, or $\mathrm{ARB}$ in the 12 months before the index date; and received a new prescription for either HCTZ, amlodipine, lisinopril, or valsartan monotherapy between 2001 and 2002. Given these criteria, the time frame for the overall analysis ranged from January 2000 to December 2003, including the pre- and post-index years. Individuals using fixed-dose combination products were excluded from the analysis. Institutional review board approval was granted from the University of Arizona Human Subjects Protection Program.

The following key variables were collected and calculated: (1) age at the index prescription date; (2) gender; (3) treatment group (ie, HCTZ, amlodipine, lisinopril, or valsartan); (4) Charlson Comorbidity Index, ${ }^{32}$ a validated measure of comorbid illness severity for each subject using International Classification of Diseases, Ninth Revision (ICD-9CM) codes, adapting the Dartmouth-Manitoba algorithm and considering the revisions of Deyo et $\mathrm{al}^{33}$ and Romano et $\mathrm{al},{ }^{34}$ with the time period for computation including the 12 months preceding the index date; and (5) concomitant utilization of antidyslipidemics, antiplatelet agents, beta-blockers, digoxin, nitrates, or warfarin. 
Outcome measures included the following: (1) persistence, defined as remaining on the index therapy and not discontinuing before the end of the 12-month post-index date, noting that discontinuation was defined as ending therapy with the index agent and not receiving another prescription for it within 60 days of exhausting the drug supply for the prior prescription (typically 90 days after the last prescription), wherein the discontinuation date is defined as the date of last dispensing; (2) time to discontinuation, defined as the number of days from dispensing the index prescription to the discontinuation date; (3) length of therapy, defined as the last prescription date for the agent within the study period minus the index prescription dispensing date; (4) medication possession ratio (MPR), defined as the percentage of time that a subject has a supply of the drug available during the 12 months after dispensing of the index prescription and including at least 2 refills (ie, the sum of the days' supply for all prescriptions dispensed during the 12 months subsequent to the index prescription divided by 365 days); and (5) adherence, defined as the sum of the days' supply of all medication dispensed (excluding the days' supply of the final prescription dispensing) divided by the length of therapy, expressed as a percentage and including at least 2 refills.

Utilization analyses were performed relative to the index date with each subject contributing 12 months of data following the index prescription. Statistical procedures used in this study included unadjusted and adjusted methods to control for covariate effects (such that the determination of statistical differences between treatment groups was valid). ${ }^{35}$ Results of unadjusted means or proportions were reported as mean \pm standard deviation or as sample size and percentage, as appropriate. Adjusted means were computed to yield the average values for MPR and adherence, controlling for the effects of age, gender, and Charlson Comorbidity Index scores; results were reported as the adjusted mean \pm SE or $95 \%$ CI.

Kaplan-Meier curves were constructed to graphically present crude survival estimates, with a logrank test for equality of survivor functions used to assess group differences. A Cox proportional hazards regression model with the Efron approximation was used to evaluate the risk of discontinuation across treatment groups, controlling for covariates including age, gender, Charlson Comorbidity In- dex, and concomitant medication use. The reference case used to gauge hazard ratios or relative risk across treatment groups was valsartan. Both graphic and statistical procedures were used to assess model diagnostics. An $\alpha$ level of 0.05 was established a priori for statistical significance testing. All analyses were performed using SAS version 9.1 (Cary, NC), STATA version 9.0 (College Station, TX), and SPSS version 14.0 (Chicago, IL).

\section{Results}

The demographic characteristics of study subjects are presented in Table 1. Overall, 60,685 records met the inclusion criteria. Mean age of the sample was $57.7 \pm 12.9$ years, and $48.2 \%$ were male. The proportion of subjects using HCTZ, amlodipine, lisinopril, and valsartan were $30.8 \%, 19.0 \%$, $34.8 \%$, and $15.4 \%$, respectively. At baseline, there were significant differences across drugs in age (by analysis of variance) and in gender (by $\chi^{2}$ ). Significantly more males than females received an initial prescription for lisinopril (males $=59.1 \%, P<$ .001 ) and valsartan (males $=53.9 \%, P<.001$ ), whereas more females than males received an initial prescription for HCTZ (males $=31.3 \%, P<.001$ ); no significant difference was noted for amlodipine according to gender. Individuals receiving amlodipine and lisinopril had higher Charlson Comorbidity Index scores than those prescribed HCTZ or valsartan, which was generally due to higher proportions of concomitant medication use (eg, antiplatelet agents, digoxin, nitroglycerin, warfarin). The number of initial prescriptions increased substantially by year, as $28 \%$ of the sample population received their initial prescription in 2001 vs $72 \%$ in 2002 .

Figure 1 shows the unadjusted 12-month persistence with the initial antihypertensive monotherapy prescription; drug utilization characteristics are reported in Table 2. Statistically significant differences in persistence across agents were observed over the duration of follow-up in the Kaplan-Meier survival curves $(P<.001$ overall). The unadjusted 1-year discontinuation rates were: valsartan (30.6\%), followed by lisinopril $(35.2 \%)$, amlodipine (39.7\%), and HCTZ (44.2\%). The unadjusted length of therapy was significantly longer for valsartan $(298.7 \pm 81.0$ days $)$, compared with lisinopril (282.0 \pm 101.0 days), amlodipine (277.8 \pm 104.9 days), or HCTZ $(265.8 \pm 109.5$ 
Table 1. Baseline Characteristics

\begin{tabular}{|c|c|c|c|c|c|}
\hline Characteristic & HCTZ & Amlodipine & Lisinopril & Valsartan & Overall \\
\hline Number of subjects & 18,713 & 11,520 & 21,138 & 9,314 & 60,685 \\
\hline (\% of sample) & $(30.8)$ & $(19.0)$ & $(34.8)$ & $(15.4)$ & $(100.0)$ \\
\hline Age, mean \pm SD & $56.6 \pm 12.9^{*}$ & $59.0 \pm 13.7^{*}$ & $57.2 \pm 12.6^{*}$ & $58.4 \pm 12.4^{*}$ & $57.7 \pm 12.9$ \\
\hline Male, n (\%) & $5,865(31.3)^{*}$ & $5,856(50.8)^{*}$ & $12,496(59.1)^{*}$ & $5,024(53.9)^{*}$ & $29,241(48.2)$ \\
\hline \multicolumn{6}{|c|}{$\begin{array}{l}\text { Charlson Comorbidity Index, } \\
\text { n (\%) }\end{array}$} \\
\hline Score $=0$ & $17,611(94.1)$ & $9,716(84.3)$ & $17,780(84.1)$ & $8,549(91.8)$ & $53,656(88.4)$ \\
\hline Score $=1$ & $564(3.0)$ & $912(7.9)$ & $1,862(8.8)$ & $417(4.5)$ & $3,755(6.2)$ \\
\hline Score $=2$ & $364(2.0)$ & $500(4.3)$ & $921(4.4)$ & $229(2.5)$ & $2,014(3.3)$ \\
\hline Score $=3$ & $95(0.5)$ & $172(1.5)$ & $331(1.6)$ & $54(0.6)$ & $652(1.1)$ \\
\hline Overall, mean $\pm \mathrm{SD}$ & $0.11 \pm 0.53^{*}$ & $0.31 \pm 0.92^{*}$ & $0.28 \pm 0.80^{*}$ & $0.15 \pm 0.63^{*}$ & $0.21 \pm 0.73$ \\
\hline \multicolumn{6}{|l|}{$\begin{array}{l}\text { Concomitant treatment, } \\
\mathrm{n}(\%)\end{array}$} \\
\hline Antidyslipidemics & $3,549(30.2)^{*}$ & $2,933(25.5)^{*}$ & $6,792(32.1)^{*}$ & $2,691(28.9)^{*}$ & $15,965(26.3)$ \\
\hline Antiplatelet agents & $456(2.4)^{*}$ & $953(8.3)^{*}$ & $1,553(7.4)^{*}$ & $365(3.9)^{*}$ & $3,327(5.5 \%)$ \\
\hline Beta-blockers & $5,648(30.2)^{*}$ & $2,741(23.8)^{*}$ & $4,876(23.1)^{*}$ & $1,460(15.7)^{*}$ & $14,725(24.3)$ \\
\hline Digoxin & $236(1.3)^{*}$ & $276(2.4)^{*}$ & $818(3.9)^{*}$ & $185(2.0)^{*}$ & $1,515(2.5)$ \\
\hline Nitroglycerin & $58(0.3)^{*}$ & $165(1.4)^{*}$ & $140(0.7)^{*}$ & $41(0.4)^{*}$ & $404(0.7)$ \\
\hline Warfarin & $470(2.5)^{*}$ & $440(3.8)^{*}$ & $1,074(5.1)^{*}$ & $319(3.4)^{*}$ & $2,303(3.8)$ \\
\hline \multicolumn{6}{|c|}{ Year of initial prescription, $\mathrm{n}^{*}$} \\
\hline 2001 & 5,754 & 3,791 & 6,578 & 1,042 & 17,165 \\
\hline 2002 & 12,959 & 7,729 & 14,560 & 8,272 & 43.520 \\
\hline
\end{tabular}

${ }^{*} P \leq .001$ between groups (one-way analysis of variance with Bonferroni post hoc for multiple comparisons or $\chi^{2}$, as appropriate). HCTZ, hydrochlorothiazide.

days) $(P<.001$ for each comparison versus valsar$\tan )$. For those who discontinued treatment, the mean time to discontinuation also was significantly longer $(P<.001)$ with valsartan $(177.5 \pm 113.8$ days) than with either amlodipine or lisinopril $(141.6 \pm 112.9$ and $141.9 \pm 110.2$ days, respectively) or HCTZ (116.2 \pm 110.0 days). After controlling for age, gender, year of initial prescription, and Charlson Comorbidity Index scores across groups, the adjusted mean MPR was highest for valsartan $(89.5 \pm 0.4 \%)$, followed by lisinopril $(84.7 \pm 0.2 \%)$, amlodipine $(78.3 \pm 0.3 \%)$, and HCTZ $(73.2 \pm 0.3 \%)(P<.001$ for each comparison versus valsartan). Adjusted adherence rates were significantly higher $(P<.001)$ for valsartan $(90.1 \pm 0.5 \%)$ and lisinopril $(89.9 \pm 0.4 \%)$, compared with either amlodipine $(85.2 \pm 0.5 \%)$ or HCTZ (78.6 $\pm 0.4 \%)$.

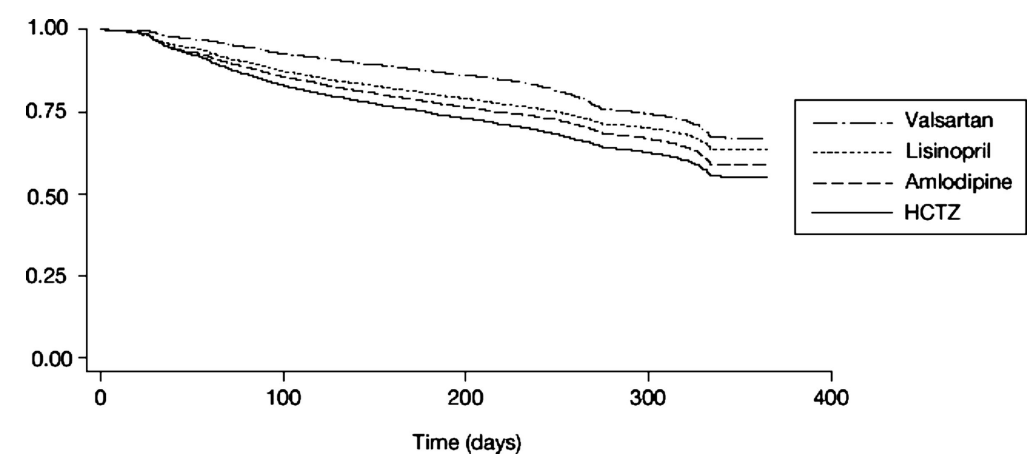

Figure 1. Kaplan-Meier estimates of the cumulative probability of discontinuation of initial antihypertensive monotherapies. There was a statistically significant difference between groups at the $P<.001$ level (log-rank test for equality of survivor functions). 
Table 2. Utilization of Antihypertensive Agents during 1 Year

\begin{tabular}{lccccc}
\hline Characteristic & $\begin{array}{c}\text { HCTZ } \\
(\mathrm{n}=18,713)\end{array}$ & $\begin{array}{c}\text { Amlodipine } \\
(\mathrm{n}=11,520)\end{array}$ & $\begin{array}{c}\text { Lisinopril } \\
(\mathrm{n}=21,138)\end{array}$ & $\begin{array}{c}\text { Valsartan } \\
(\mathrm{n}=9314)\end{array}$ & $\begin{array}{c}\text { Overall } \\
(\mathrm{n}=60,685)\end{array}$ \\
\hline Persistence, \% & $55.8^{*}$ & $60.3^{*}$ & $64.8^{*}$ & $69.4^{*}$ & 61.9 \\
Discontinuation, \% & $44.2^{*}$ & $39.7^{*}$ & $35.2^{*}$ & $30.6^{*}$ & 38.1 \\
Length of therapy, days & $265.8^{*}$ & $277.8^{*}$ & $282.0^{*}$ & $298.7^{*}$ & 278.8 \\
$\quad[$ Mean (95\% CI)] & $(264.2-267.3)$ & $(175.8-279.7)$ & $(280.7-283.4)$ & $(297.1-300.4)$ & $(277.9-279.6)$ \\
Time to discontinuation, days $\dagger$ & $116.2^{*}$ & $141.6^{*}$ & $141.9^{*}$ & $177.5^{*}$ & 137.0 \\
$\quad[$ Mean (95\% CI)] & $(113.9-118.6)$ & $(138.3-144.9)$ & $(139.4-144.4)$ & $(173.4-181.5)$ & $(135.6-138.5)$ \\
MPR, adjusted \% & $73.2 \neq$ & $78.3 \neq$ & $84.7 \neq$ & $89.5 \neq$ & 81.6 \\
$\quad(95 \%$ CI) & $(72.7-73.6)$ & $(77.7-79.0)$ & $(84.3-85.2)$ & $(88.8-90.2)$ & $(81.3-81.9)$ \\
Adherence, adjusted \% & $78.6 \neq$ & $85.2 \ddagger$ & $89.9 \neq$ & $90.1 \neq$ & 86.2 \\
$\quad(95 \%$ CI) & $(77.9-79.4)$ & $(84.3-86.1)$ & $(89.3-90.6)$ & $(89.0-91.1)$ & $(85.8-86.6)$ \\
\hline
\end{tabular}

${ }^{*} P \leq .001$ between groups (one-way analysis of variance with Bonferroni post-hoc for multiple comparisons or $\chi^{2}$ as appropriate). $\dagger$ Computed for subjects discontinuing therapy.

‡ Overall model statistically significant at $P \leq .001$; adjustment included baseline age, gender, year of initial prescription, and Charlson comorbidity score.

HCTZ, hydrochlorothiazide; CI, confidence interval; MPR, medication possession ratio.

Table 3 shows results of the Cox proportional hazards regression model using treatment discontinuation as the outcome variable, and controlling for age, gender, year of initial prescription, and Charlson Comorbidity Index scores. Relative to valsartan, individuals who received HCTZ, amlodipine, or lisinopril were $53 \%, 32 \%$, and $14 \%$ more likely to discontinue therapy, respectively (all $P<.001)$. Using HCTZ as the standard, amlodip- ine, lisinopril, and valsartan were 14\%, 26\%, and $35 \%$ less likely to discontinue therapy, respectively (all $P<.001)$. Women $(P<.008)$, older persons $(P<.001)$, and those with higher Charlson Comorbidity Index scores $(P<.001)$ also were significantly more likely to discontinue treatment. After controlling for other covariates, first starts in the year 2002 were associated with a slightly (but statistically significantly) lower risk of discontinuation

Table 3. Results of Cox Regression Model for Drug Discontinuation, Controlling for Demographics, Year of Initial Prescription (2001 as Referent), and Concomitant Treatment Regimens*

\begin{tabular}{lcr}
\hline Variable & $\begin{array}{c}\text { Hazard Ratio } \\
(95 \% \text { CI })\end{array}$ & $\begin{array}{c}\text { Value of } P \\
\text { between Groups }\end{array}$ \\
\hline Demographics & & $<.001$ \\
$\quad$ Age & $0.99(0.99-0.99)$ & .008 \\
Gender (male) & $0.95(0.93-0.99)$ & $<.001$ \\
Year of initial prescription (vs. 2002) & $0.91(0.88-0.94)$ & $<.001$ \\
Charlson Comorbidity Index & $1.17(1.15-1.20)$ & $<.001$ \\
Treatment ${ }^{\dagger}$ & & $<.001$ \\
Hydrochlorothiazide & $1.53(1.47-1.61)$ & $<.001$ \\
Amlodipine & $1.32(1.26-1.39)$ & $<.001$ \\
Lisinopril & $1.14(1.09-1.20)$ & .017 \\
Concomitant Treatment Regimens & & .009 \\
Antidyslipidemics & $0.88(0.85-0.91)$ & $<.001$ \\
Antiplatelet agents & $1.08(1.01-1.15)$ & $<.001$ \\
Beta-blockers & $0.96(0.92-0.99)$ & .167 \\
Digoxin & $1.18(1.07-1.29)$ & \\
Nitroglycerin & $1.35(1.16-1.58)$ & \\
Warfarin & $1.05(0.98-1.14)$ & \\
\hline
\end{tabular}

* Cox proportional hazards model with Efron approximation method.

$\dagger$ Referent treatment $=$ valsartan. 
(relative to 2001, $P<.001$ ). Similar results were obtained in an extended model that controlled for concomitant medication use. However, utilization of antiplatelet agents, digoxin, and nitroglycerin was associated with a higher risk of discontinuation, whereas use of either antidyslipidemic agents or beta-blockers was associated with a significantly lower risk of discontinuation. A post hoc Cox regression analysis for individuals 65 years of age or older $(\mathrm{n}=15,172)$ indicated that the relative risk of discontinuation for HCTZ, amlodipine, or lisinopril was $50 \%, 24 \%$, or $20 \%$ higher, respectively, than for valsartan (all comparisons $P<.001$ ). In this analysis, neither gender nor concomitant treatment was significantly associated with discontinuation of the 4 medications investigated.

\section{Discussion}

This large-scale analysis of drug utilization patterns within the United States found that 31\% to $44 \%$ of treatment-naive subjects initiating therapy with commonly prescribed antihypertensive monotherapies actually utilized no therapy for at least 2 months during the first year of treatment. Despite this considerable gap, other measures of drug utilization, including MPR and adherence, seemed to be more favorable, ranging from $73 \%$ to $90 \%$. The patterns of drug utilization differed by drug class. The ARB, valsartan, was associated with significantly improved measures of persistence, length of therapy, time to discontinuation, MPR, and risk of discontinuation, compared with representatives of the thiazide diuretic (HCTZ), CCB (amlodipine), or ACE inhibitor (lisinopril) drug classes. Adherence was highest for valsartan and lisinopril, compared with HCTZ and amlodipine.

Prior research has examined nonadherence to drug treatment regimens over a range of medical conditions. It has been reported that $20 \%$ to $60 \%$ of individuals improperly cease taking medication and that $20 \%$ to $80 \%$ fail to take their medications appropriately. ${ }^{36}$ In the most recent National Health and Nutrition Examination Survey (2001 to 2002), $71 \%$ of hypertensive Americans were aware of their diagnosis, but only $61 \%$ reported taking antihypertensive medication. ${ }^{37}$ Furthermore, only approximately one-third of all hypertensive persons achieved a blood pressure goal below 140/90 mm $\mathrm{Hg}$. In a nationally representative population sample during 1996, physician compliance with pre- scribing guidelines of the Fifth Joint National Committee on Detection, Evaluation and Treatment of High Blood Pressure (JNC V) was only $37 \%$ for first-line antihypertensive drugs and $67 \%$ for second-line drugs. ${ }^{38}$

The findings of this study are consistent with prior research suggesting that ARBs are associated with significantly higher persistence rates than other commonly used antihypertensive drug classes. ${ }^{19-24}$ This conclusion seems to be consistent across a wide range of settings and patient populations: in managed care organizations ${ }^{19,21}$ and general clinical pharmacy practice, ${ }^{20,22,23}$ in the United States, ${ }^{19,21}$ Canada, ${ }^{20,23}$ and Europe,${ }^{22,24}$ in newly diagnosed hypertensive patients naive to drug therapy, ${ }^{19,20,22,24}$ and in those who have other conditions, including previously treated hypertension, whether observed over the short (1 year) ${ }^{19,24}$ or longer term (up to 4 years). ${ }^{21,23}$

In all these studies, as in the current analysis, the rank order of antihypertensive drugs regarding long-term persistence (from most to least favorable) was $\mathrm{ARB}>\mathrm{ACE}$ inhibitor $\geq \mathrm{CCB}>$ diuretic. For example, approximately two-thirds of patients in managed care and usual-care settings continued on ARB therapy over 1 year, compared with $50 \%$ to $58 \%$ of patients taking an ACE inhibitor, $50 \%$ to $53 \%$ with a CCB, and only $38 \%$ with a diuretic. ${ }^{19,39}$ Over a 4 -year period, during which time adherence to antihypertensive therapy declines, persistence remained at approximately $51 \%$ with an $\mathrm{ARB}$, versus $47 \%$ with an ACE inhibitor, $41 \%$ with a CCB, and only $16 \%$ with a diuretic. ${ }^{21}$ Examining comparative persistence, adherence, and discontinuation rates within real-world settings, the current analyses are consistent with clinical trial findings that ARBs are associated with the fewest adverse effects among the most commonly utilized antihypertensive drug classes. ${ }^{12,17,39,40}$ In addition to the potential for improved cardiovascular and renal outcomes, persistence or continuous use of antihypertensive therapy has been associated with lower overall health care costs. ${ }^{30}$

The data in the current study differ somewhat from those used in other reports. The database queried in the current investigation contains many more unique subject records than previous databases, thus increasing the statistical power of the analyses. The database contains extensive information regarding other diagnoses with which a Charlson Comorbidity Index score was calculated. This 
risk adjustment measure was used to control statistically for various case-mix across treatment groups. The more extensive information available in this database also allowed us to conduct a secondary analysis that controlled for concomitant medication use (as a marker of disease severity), which further standardized the groups regarding case-mix differences. The methods used in this investigation and the consistency of our results and those from prior studies support the validity of these findings.

Our analyses have a number of limitations, particularly given the retrospective design and the potential for confounding by indication bias. ${ }^{41,42}$ The large sample size could have generated statistically significant results that may have little clinical or practical importance. No information regarding drug-related adverse events and other potential factors that may influence the selection of an individual antihypertensive agent was available within the administrative claims database. Characteristics of the health care provider prescribing the drug, possible differences in copayment by patients across therapies, and availability of agents on specific formularies, factors that may influence both prescribing practices and copayments, were unavailable. No information exists in the database about individuals who may have received an initial sample of antihypertensive agent in a clinic setting, but who never filled a prescription for the sample drug. This study population included only subjects with medical and pharmacy benefit coverage, and thus did not capture the approximately one third of Americans who used pharmacy services but had no pharmacy benefit plan in 2001 to 2002. Therefore, these findings may not be generalizable to all health care systems or pharmacy payment structures. Consistent with all studies of prescription databases, refill history is a proxy for persistence and adherence, as there is a lack of assurance that a filled prescription was taken as directed. Empiric evidence suggests, however, a strong correlation between pharmacy claims and drug exposure. ${ }^{43}$

Overall, this investigation of real-world patterns of drug utilization found a relatively high degree of treatment gaps and differences in adherence measures among the four antihypertensive agents studied. Based on these findings, health care providers and organizations should consider evaluating their specific populations to identify and rectify patterns of suboptimal drug utilization. Although assessing the causes or effects of poor adherence was not part of this investigation, further research is needed to examine factors and outcomes associated with treatment discontinuation such as cost, access, adverse effects, treatment failures, and social and behavioral components. Further analyses concerning physician compliance with clinical practice guidelines such as JNC 7 are also recommended.

\section{Conclusion}

This investigation of real-world drug utilization patterns among 60,685 treatment-naive subjects within the United States indicates that approximately one third to one half of individuals discontinued use of commonly prescribed antihypertensive agents for 60 days or more within 1 year after treatment initiation. Among the agents evaluated, valsartan had higher persistence and adherence patterns, compared with HCTZ, amlodipine, or lisinopril. The observed differences in risk of discontinuation among medications studied may be illustrative of broader relationships among effectiveness, tolerability of medications, subjects' drug utilization behavior, and the selection of a specific antihypertensive drug. Higher pharmacy refill rates may correlate with better blood pressure control, which has been linked directly to a reduced longterm risk of cardiovascular events. ${ }^{15,44}$ The public health concerns surrounding suboptimal patient adherence to antihypertensive therapy places increased responsibility on clinicians and health care systems to evaluate drug utilization patterns to identify patterns of poor persistence and a higher risk of discontinuation within their respective populations.

\section{References}

1. Prospective Studies Collaboration. Age-specific relevance of usual blood pressure to vascular mortality: meta-analysis of individual data for one million adults in 61 prospective studies. Lancet 2002;360: 1903-13.

2. American Heart Association. Heart Disease and Stroke Statistics: 2006 Update. Dallas, TX: American Heart Association, 2005.

3. Ezzati M, Lopez AD, Rodgers A, Vander Hoorn S, Murray CJL, and the Comparative Risk Assessment Collaborating Group. Selected major risk factors and global and regional burden of disease. Lancet 2002; 360:1347-60.

4. Fields LE, Burt VL, Cutler JA, et al. The burden of 
adult hypertension in the United States 1999 to 2000: A rising tide. Hypertension 2004;44:398-404.

5. Kearney PM, Whelton M, Reynolds K, et al. Global burden of hypertension: analysis of worldwide data. Lancet 2005;365:217-23.

6. Blood Pressure Lowering Treatment Trialists' Collaboration. Effects of different blood-pressure-lowering regimens on major cardiovascular events: results of prospectively-designed overviews of randomised trials. Lancet 2003;362:1527-35.

7. Staessen JA, Wang J-G, Thijs L. Cardiovascular prevention and blood pressure reduction: a quantitative overview updated until 1 March 2003. J Hypertens 2003;21:1055-76.

8. Chobanian AV, Bakris GL, Black HR, et al. Seventh report of the Joint National Committee on Prevention, Detection, Evaluation, and Treatment of High Blood Pressure. Hypertension 2004;42:1206-52.

9. Carol JJ, Salas M, Speckman JL, Raggio G, Jackson JD. Persistence with treatment for hypertension in actual practice. CMAJ 1999;160:31-7.

10. Degli Esposti L, Degli Esposti E, Valpiani G, et al. A retrospective, population-based analysis of persistence with antihypertensive drug therapy in primary care practice in Italy. Clin Ther 2002;24:1347-57.

11. Gregoire JP, Moisan J, Guibert R, et al. Determinants of discontinuation of new courses of antihypertensive medications. J Clin Epidemiol 2002;55: 728-35.

12. Black HR, Graff A, Shute D, et al. Valsartan, a new angiotensin II antagonist for the treatment of essential hypertension: efficacy, tolerability and safety compared to an angiotensin-converting enzyme inhibitor, lisinopril. J Hum Hypertens 1997;11:483-9.

13. Bremner AD, Baur M, Oddou-Stock P, Bodin F. Valsartan: long-term efficacy and tolerability compared to lisinopril in elderly patients with essential hypertension. Clin Exp Hypertens 1997;19:126385.

14. Corea L, Cardoni O, Fogari R, et al. Valsartan, a new angiotensin II antagonist for the treatment of essential hypertension: a comparative study of the efficacy and safety against amlodipine. Clin Pharmacol Ther 1996;60:341-6.

15. Julius S, Kjeldsen SE, Weber M, et al., for the VALUE trial group. Outcomes in hypertensive patients at high cardiovascular risk treated with regimens based on valsartan or amlodipine: the VALUE randomised trial. Lancet 2004;363:2022-31.

16. Malacco E, Santonastaso M, Vari NA, et al. Comparison of valsartan $160 \mathrm{mg}$ with lisinopril $20 \mathrm{mg}$, given as monotherapy or in combination with a diuretic, for the treatment of hypertension: the Blood Pressure Reduction and Tolerability of Valsartan in Comparison with Lisinopril (PREVAIL) study. Clin Ther 2004;26:855-65.

17. Malacco E, Varì N, Capuano V, et al. for the ValSyst Investigators. A randomized, double-blind, ac- tive-controlled, parallel-group comparison of valsartan and amlodipine in the treatment of isolated systolic hypertension in elderly patients: The ValSyst study. Clin Ther 2003;25:2765-80.

18. Rizzo JA, Simons WR. Variations in compliance among hypertensive patients by drug class: Implications for health care costs. Clin Ther 1997;19:144657.

19. Bloom BS. Continuation of initial antihypertensive medication after 1 year of therapy. Clin Ther 1998; 20:671-81.

20. Caro JJ, Speckman JL, Salas M, Raggio G, Jackson JD. Effect of initial drug choice on persistence with antihypertensive therapy: The importance of actual practice data. CMAJ 1999;160:41-6.

21. Conlin PR, Gerth WC, Fox J, Roehm JB, Boccuzzi SJ. Four-year persistence patterns among patients initiating therapy with the angiotensin II receptor antagonist losartan versus other antihypertensive drug classes. Clin Ther 2001;23:1999-2010.

22. Degli Esposti E, Sturani A, Di Martino M, et al. Long-term persistence with antihypertensive drugs in new patients. J Hum Hypertens 2002;16:439-44.

23. Marentette MA, Gerth WC, Billings DK, Zarnke KB. Antihypertensive persistence and drug class. Can J Cardiol 2002;18:649-56.

24. Hasford J, Mimran A, Simons WR. A populationbased European cohort study of persistence in newly diagnosed hypertensive patients. J Hum Hypertens 2002;16:569-75.

25. The ALLHAT Officers and Coordinators for the ALLHAT Collaborative Research Group. Major cardiovascular events in hypertensive patients randomized to doxazosin vs chlorthalidone: the Antihypertensive and Lipid-Lowering Treatment to Prevent Heart Attack Trial (ALLHAT). JAMA 2000; 283:1967-75.

26. The ALLHAT Officers and Coordinators for the ALLHAT Collaborative Research Group. Major outcomes in high-risk hypertensive patients randomized to angiotensin-converting enzyme inhibitor or calcium channel blocker vs diuretic: the Antihypertensive and Lipid-Lowering Treatment to Prevent Heart Attack Trial (ALLHAT). JAMA 2002;288: 2981-97.

27. Esposti LD, Di Martino M, Saragoni S, et al. Pharmacoeconomics of antihypertensive drug treatment: an analysis of how long patients remain on various antihypertensive therapies. J Clin Hypertens (Greenwich) 2004;6:76-84.

28. Elliott WJ. Compliance-and improving it-in hypertension. Manag Care 2003;12(8 Suppl):56-61.

29. Skrepnek GH. Cost-effectiveness analysis. In: Bootman JL, Townsend RJ, McGhan WF, eds. Principles of Pharmacoeconomics. Third edition. Cincinnati, OH: Harvey Whitney Books, 2005.

30. McCombs JS, Nichol MB, Newman CM, Sclar DA. The costs of interrupting antihypertensive drug 
therapy in a Medicaid population. Med Care 1994; 32:214-26.

31. The Top 300 Prescriptions for 2004 by Number of US Prescriptions Dispensed. Available at: www. rxlist.com/top200.htm. Accessed March 27, 2006.

32. Charlson ME, Pompei P, Ales KL, MacKenzie CR. A new method of classifying prognostic comorbidity in longitudinal studies: development and validation. J Chron Dis 1987;40:373-83.

33. Deyo RA, Cherkin DC, Ciol MA. Adapting a clinical comorbidity index for use with ICD-9-CM administrative databases. J Clin Epidemiol 1992;45:613-9.

34. Romano PS, Roos LL, Jollis JG. Adapting a clinical comorbidity index for use with ICD-9-CM administrative data: Differing perspectives. J Clin Epidemiol 1993;46:1075-9.

35. Skrepnek GH. Regression methods in the empiric analysis of health care data. J Manag Care Pharm 2005;11:240-51.

36. Burke LE, Ockene IS, ed. Compliance in Health Care and Research. New York: Futura Publishing, 2001.

37. Cheung BM, Ong KL, Man YB, Lam KS, Lau CP. Prevalence, awareness, treatment, and control of hypertension: United States National Health and Nutrition Examination Survey 2001-2002. J Clin Hypertens (Greenwich) 2006;8:93-8.
38. Xu KT, Moloney M, Phillips S. Economics of suboptimal drug use: cost-savings of using JNC-recommended medications for the management of uncomplicated essential hypertension. Am J Manag Care 2003;9:529-36.

39. Wogen J, Kreilick CA, Livornese RC, Yokoyama K, Frech F. Patient adherence with amlodipine, lisinopril, or valsartan therapy in a usual-care setting. J Manag Care Pharm 2003;9:424-9.

40. Wu SC, Liu CP, Chiang HT, Lin SL. Prospective and randomized study of the antihypertensive effect and tolerability of three antihypertensive agents, losartan, amlodipine, and lisinopril, in hypertensive patients. Heart Vessels 2004;19:13-18.

41. Gerth WC. Compliance and persistence with newer antihypertensive agents. Curr Hypertens Rep 2002; 4:424-33.

42. Spence JD, Hurley TC, Spence JD. Actual practice in hypertension: Implications for persistence with and effectiveness of therapy. Curr Hypertens Rep 2001;3:481-7.

43. Elliott WJ. Compliance strategies. Curr Opin Nephrol Hypertens 1994;3:271-8.

44. Neutel JM, Smith DH. Improving patient compliance: A major goal in the management of hypertension. J Clin Hypertens (Greenwich) 2003;5:127-32. 